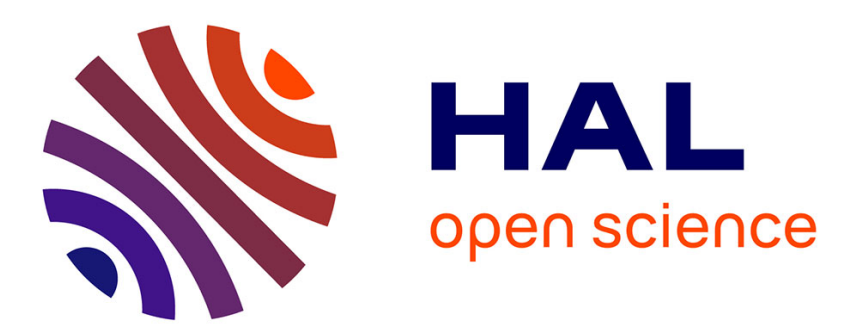

\title{
Time-dependent analysis of the mechanism for two-photon double escape in helium: from very long to attosecond time scales
}

E Foumouo, A Hamido, Ph Antoine, B Piraux, H Bachau, R Shakeshaft

\section{- To cite this version:}

E Foumouo, A Hamido, Ph Antoine, B Piraux, H Bachau, et al.. Time-dependent analysis of the mechanism for two-photon double escape in helium: from very long to attosecond time scales. Journal of Physics B: Atomic, Molecular and Optical Physics, 2010, 43 (9), pp.91001. 10.1088/09534075/43/9/091001 . hal-00569913

\section{HAL Id: hal-00569913 \\ https://hal.science/hal-00569913}

Submitted on 25 Feb 2011

HAL is a multi-disciplinary open access archive for the deposit and dissemination of scientific research documents, whether they are published or not. The documents may come from teaching and research institutions in France or abroad, or from public or private research centers.
L'archive ouverte pluridisciplinaire $\mathbf{H A L}$, est destinée au dépôt et à la diffusion de documents scientifiques de niveau recherche, publiés ou non, émanant des établissements d'enseignement et de recherche français ou étrangers, des laboratoires publics ou privés. 


\title{
Time-dependent analysis of the mechanism for two-photon double-escape in helium: from very long to attosecond time scales
}

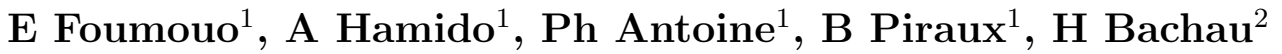 \\ and \\ R Shakeshaft ${ }^{3}$ \\ ${ }^{1}$ Institute of Condensed Matter and Nanosciences, Université catholique de Louvain, \\ 2, chemin du Cyclotron, B-1348 Louvain-la-Neuve, Belgium \\ ${ }^{2}$ Centre des Lasers Intenses et Applications (CELIA), UMR 5107 de l'Université de \\ Bordeaux 1-CNRS-CEA, 351 Cours de la Libération, F-33405 Talence, France \\ ${ }^{3}$ Physics Department, University of Southern California, Los Angeles, CA \\ 90089-0484, USA
}

\begin{abstract}
We consider the non-sequential double-ionization of helium by two XUV photons. To gain further insight into the double escape mechanism, we examine the interaction of helium with attosecond pulses. Such an interaction allows us to unveil the timescale on which the two electrons interact. By analyzing the behaviour of the electron angular and energy distributions versus the pulse duration, we show that in the limit of ultrashort pulse durations, it becomes possible to disentangle the process in which each electron absorbs one photon from the process in which one electron absorbs both photons while ejecting the other electron by a collision. These results, which confirm the double escape mechanism that we had proposed earlier (J. Phys. B 41051001 (2008)), are obtained using two different time-dependent theoretical approaches. The first one is based on Jacobi matrix calculations to extract the relevant information from the ionized wavepacket. In the second one, we project the final wavepacket on two Coulomb functions. We also develop a simple model based on time-dependent perturbation theory.
\end{abstract}

Submitted to: J. Phys. B: At. Mol. Opt. Phys. as a Fast Track Communication 


\section{Introduction}

Double-ionization of helium by absorption of one XUV photon has been investigated experimentally in great detail $[1,2]$, and is now well understood. However, doubleionization of helium by two-photon absorption, currently a subject of much interest, as yet lacks a complete description, particularly in the energy regime where only nonsequential ionization is possible. The inclusion of an additional photon opens up a rich variety of new phenomena; but it also introduces serious theoretical and experimental complications $[3,4,5,6,7,8,9,10,11,12,13,14,15]$.

Two-photon double-ionization (TPDI) of helium is energetically allowed at photon energies above $39.5 \mathrm{eV}$. For photon energies above $54.4 \mathrm{eV}$, sequential ionization can take place, where the photons are absorbed at different times; first one photon ejects an electron, the helium ion relaxes, and subsequently another photon ejects the remaining electron. Our focus is on the energy regime 39.5-54.4 eV, where the sequential process is energetically forbidden. In this regime TPDI is direct, and the absorption of the second photon must occur within a time smaller than the relaxation time of the residual ion. In a previous paper [16] we showed that electron angular correlation strongly favours back-to-back electron emission along the polarization axis while dynamical screening, i.e. radial correlation, leads to an equipartition of the electron energy for a broad range of photon energies from the TPDI threshold to about $48 \mathrm{eV}$. This mechanism reveals itself in the recoil-ion momentum distribution, which should be peaked around zero. The recent experimental results of Rudenko et al. [14] obtained in helium and neon with a reaction microscope at FLASH (the free-electron laser at Hamburg) seem to confirm these features although the statistics as well as the resolution are still rather poor.

In the present paper, we expand upon this mechanism. We provide an in-depth analysis of electronic correlations during the double-escape process, and we show that the role of dynamical screening depends very much on the pulse duration. First, we consider long pulses, where the notion of a photon is meaningful and where, in the direct ionization regime, the notion of a generalized cross section is also meaningful. Then we consider the attosecond (as) pulse regime where the notion of neither a photon nor a generalized cross section make sense. Finally, we show that for ultrashort pulses, it is possible to make an approximate analytic description of the process. In principle, attosecond pulses can probe the correlated electron motion on the attosecond time scale. Indeed, our results for the angular and energy distributions clearly demonstrate that the atom absorbs energy from the field in a time interval that is shorter than the time scale associated to the electron correlations. In the limit of ultrashort pulse durations, it becomes possible to distinguish the process in which each electron absorbs a "photon" from the process in which one electron absorbs both "photons" and ejects the other electron by a collision. We confirm this within a simple model based on lowest-order time-dependent perturbation theory.

As noted above, TPDI is not straightforward to investigate either experimentally or theoretically. From the experimental point of view, the fact that two-photon absorption 
is a non-linear process implies that its probability to occur is low and therefore XUV sources that are more intense than the synchrotron ones are required. At the present stage, the only available sources $[18,17]$ are high order harmonic generation (HOHG) and the free electron laser (FEL). Attosecond pulses are currently produced in the laboratory by using $\mathrm{HOHG}$, but for the time being their intensity is too low to perform the type of experiment we envisage, although it is reasonable to believe that, given the fast progress in the field, such experiments will be possible in the near future. Another problem is that the characterization of the pulses (temporal and spatial profiles) emitted by the HOHG and FEL sources is still very difficult to obtain and not even known in some cases. This and the fact that, in addition to the non-sequential process, sequential processes involving three photons contribute appreciably to the cross section for doubleionization [13], put the absolute measurement of the total generalized cross section for two-photon double-ionization (TPDI) of helium presently out of reach.

On the theoretical side, the duration and the estimated peak intensity of the pulses provided by the new HOHG and FEL sources are such that TPDI can be treated within the framework of lowest non-vanishing order of perturbation theory. Despite this simplification the calculation is still a difficult one since it involves an infinite summation on all the intermediate P-states as well as some information about the final double continuum wavefunction whose asymptotic behaviour is forbidding. It is therefore not surprizing that there is presently no consensus among theorists as to which calculations $[3,5,6,9,12,15,4,7,8,10]$ yield correct results. The approach adopted here consists in solving the time-dependent Schrödinger equation to generate the final two-electron wavepacket after the interaction with the pulse [3, 5, 6, 9, 12, 15].

\section{Two-photon double-ionization regimes}

To put the following sections in perspective we distinguish different regimes by the duration of the pulse. In each regime the dynamics of TPDI is different.

In the long-pulse regime the frequency bandwidth $\Delta \omega$ is much smaller than the frequency $\omega$, so the pulse duration is of the order of ten cycles or more. In this regime we can distinguish two subregimes by the photon energy. In the range of photon energies $39.5 \mathrm{eV}$ (the TPDI threshold) to $54.4 \mathrm{eV}$ (the ionization potential of $\mathrm{He}^{+}$) only direct double ionization occurs; in the range beyond this, TPDI is predominantly sequential.

When TPDI is sequential the process is dominated by transition channels that require no interaction between the electrons [19]. This leads to an electron energy distribution that exibits two sharp peaks, one associated with single ionization of He following absorption of the first photon, the other associated with ionization of $\mathrm{He}^{+}$following absorption of the second photon. The two peaks are separated by the "dielectronic interaction energy" $E_{d i}$. This energy is $29.9 \mathrm{eV}$, the difference between the exact ground-state energy of He and the approximate ground state energy obtained within the hydrogenic model where each electron moves independently of, and unscreened by, the other. In the sequential process the dielectronic interaction energy 
is transferred to the electron that is first ejected while the residual $\mathrm{He}^{+}$relaxes to its ground state; the characteristic relaxation time is $\tau_{d i} \equiv 2 \pi / E_{d i}$. For sufficiently low field intensities, i.e. in the absence of significant depletion of ground-state population, the probability of sequential TPDI is proportional to the square of the pulse duration, so the notion of a generalized cross section has no meaning. Indeed, the ionization rate is time-dependent and a proper quantitative description of the sequential TPDI process requires the solution of two rate equations [20].

When TPDI is direct, as it is for photon energies between $39.5 \mathrm{eV}$ and $54.4 \mathrm{eV}$, the second photon is absorbed before the residual ion has time to relax. In this case the dielectronic interaction energy $E_{d i}$ is shared by both electrons and the process is governed by electron correlations. The probability for direct TPDI is proportional to the pulse duration, so a generalized cross section can be defined.

In the ultrashort pulse regime we call transient, the pulse duration is of the order of or less than the characteristic relaxation time $\tau_{d i}$. Note that for helium, $\tau_{d i}$ is about 140 as. Hence, for the frequencies considered here, the notion of a photon looses its meaning since the frequency bandwidth is of the same order as the frequency itself. In this case, irrespective of the value of the averaged photon energy, the TPDI process is neither direct nor sequential [21, 22]. In fact, the ionization yield varies strongly and certainly not linearly or quadratically with the pulse duration.

\section{Non-sequential TPDI in the long-pulse regime}

By analyzing the electron angular and energy distributions in the long-pulse regime, Foumouo et al. [16] proposed the following double escape mechanism: both electrons are predominantly emitted back to back along the polarization axis. In addition, equal energy sharing is strongly favoured for photon energies ranging from threshold up to about $48 \mathrm{eV}$, thereby indicating that dynamical screening, i.e. radial correlations, is essential for both electrons to escape. Above a photon energy of $48 \mathrm{eV}$ energy sharing is no longer critical: each electron can escape independently of the other. In order to demonstrate the role of dynamical screening, we show in Fig.1, snapshots of the radial doubly ionized wave packet at several instants after the end of the interaction of the helium system with a sine-squared pulse whose photon energy and peak intensity are $42 \mathrm{eV}$ and $10^{13} \mathrm{Watt} / \mathrm{cm}^{2}$, respectively. We consider the configuration in which the electrons are emitted back-to-back along the polarization axis; hence the electron probability distribution is symmetric about the line $r_{1}=r_{2}$ where $r_{1}$ and $r_{2}$ are the distances of the electrons from the nucleus. The doubly ionized wave packet is obtained by subtracting from the total wave packet, the bound-state and singlecontinuum contributions. The latter one is calculated by means of the J-matrix method which is described in detail in Foumouo et al. [6] and summarized in Section 4 of this contribution. Immediately after the interaction with the pulse is over (Fig. 1a) the radial probability density is concentrated at small electron-nucleus distances. At 4 optical periods after the end of the interaction with the pulse (Fig. 1b) we see that 

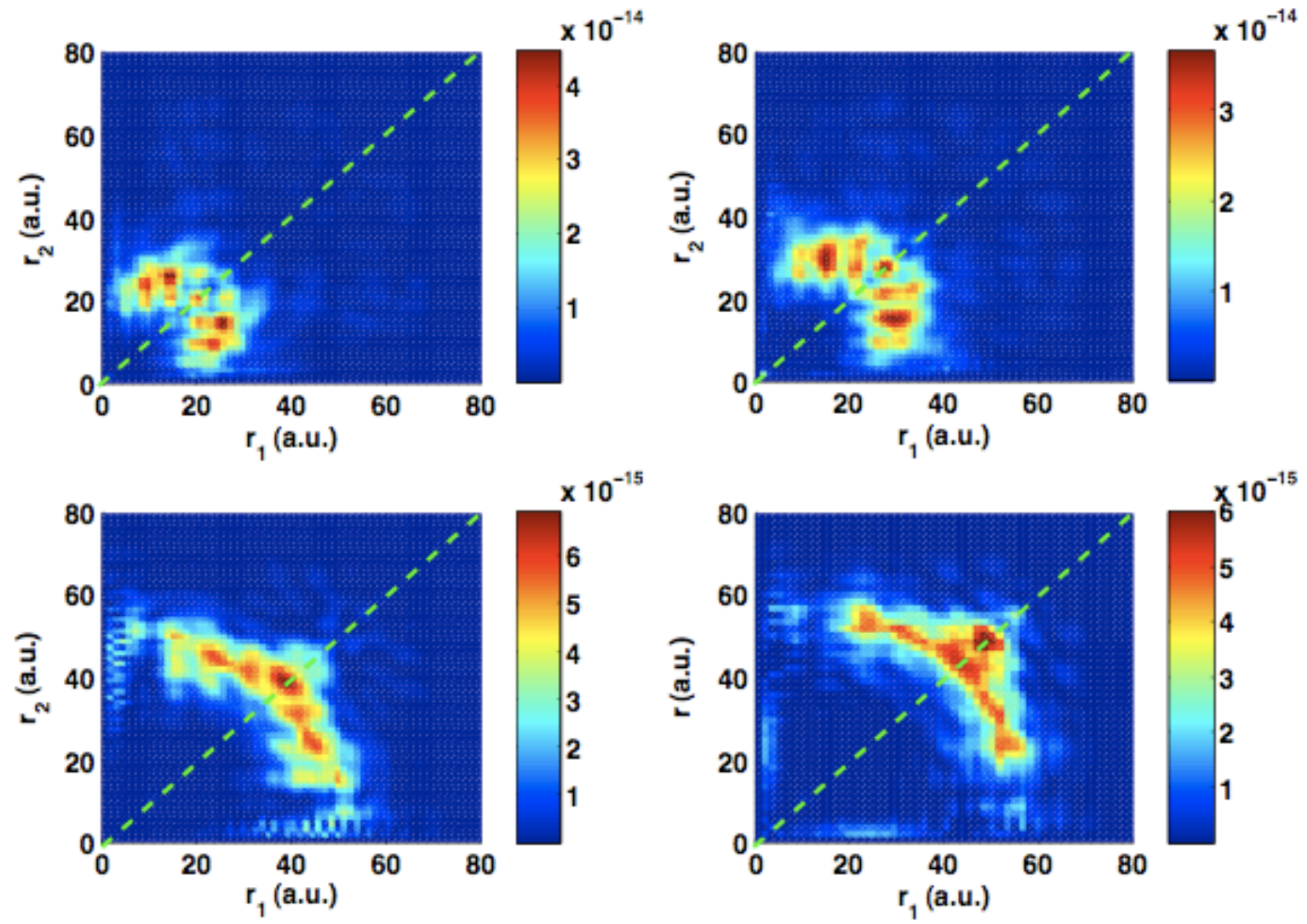

Figure 1. Snapshots of the probability density associated to the radial doubly ionized wave packet at different instants after the pulse has ceased to interact with the atomic system. The pulse has a sine squared profile, its duration is 10 optical periods, the photon energy is $42 \mathrm{eV}$, and the peak intensity is $10^{13} \mathrm{Watt} / \mathrm{cm}^{2}$. The snapshots are taken at (a) 0, (b) 4, (c) 7, and (d) 10 optical periods after the atom-pulse interaction has vanished. The snapshots are shown for the configuration in which the electrons are ejected back-to-back. The dashed line is the $r_{1}=r_{2}$ line.

the probability density has begun to move away from the nucleus. A small fraction has collected on the line $r_{1}=r_{2}$ while two "wings" on each side of this line have started to develop. At 7 optical periods (Fig. 1c) and 10 optical periods (Fig. 1d) after the end of the interaction with the pulse, we see that the probability density is mainly concentrated along the line $r_{1}=r_{2}$ while the "wings" have become thinner. This behaviour of the probability density is a clear signature of dynamical screening. Each electron experiences a different effective charge for the nucleus because of mutual screening. Indeed, assuming that the faster electron is created in the continuum closer to the nucleus than the slower one, the faster electron has to overcome a stronger Coulomb attraction with the nucleus and hence suffers a more severe slowdown than the other electron. After a while, both 
electrons have roughly the same energy and are at similar distances from the nucleus. When this happens, the effective charge of the nucleus is the same for both electrons and the screening becomes static. A simple calculation shows that if we treat the motion of the electrons along the polarization axis as classical, the static effective charge [23] is equal to 1.75. The fact that this effective charge is less than 2 clearly shows that the electron-electron interaction, whose strength is of the same order as the electronnucleus interaction, cannot be neglected even at very large distances. We have checked our quantum calculations by performing classical one-dimensional simulations based on solving Newton's equations. These simulations lead to similar conclusions; in particular, if the slower electron is created in the continuum closer to the nucleus than the faster one, its probability to recombine with the nucleus is very high, in agreement with the predictions of Rau [24].

\section{Electron angular and energy distributions in the attosecond pulse regime}

In this section, we consider the interaction of helium with a few-cycle pulse. The electric field is defined as follows:

$$
\vec{E}(t)=E_{0} f(t) \sin (\omega t+\phi) \vec{e}_{z},
$$

where $\vec{e}_{z}$ is the unit vector along the polarization axis, $\omega$ and $\phi$ are the frequency and the phase, respectively, and the envelope $f(t)$ is

$$
\begin{aligned}
f(t) & =\cos ^{2}(t / \tau), & & |t| \leq \pi \frac{\tau}{2}, \\
& =0, & & |t|>\pi \frac{\tau}{2} .
\end{aligned}
$$

In this expression, $\pi \tau$ represents the total duration of the pulse expressed as an integer number of optical periods. It is easy to check that:

$$
\int_{-\pi(\tau / 2)}^{\pi(\tau / 2)} \mathrm{d} t \vec{E}(t)=0
$$

for any pulse duration and phase. This means that even for few-cycle pulses, there is no static field component, so no problems related to the gauge invariance arise. Moreover, the spectral width $\Delta \omega$ of such a pulse, defined as the full width at half maximum of the square of the Fourier transform of $\vec{E}(t)$, is given by $1.44 \omega / n$ where the integer $n$ gives the total number of optical cycles within the pulse. In the cases treated here, $n$ is as small as 2 so that $\Delta \omega$ is of the order of $\omega$. The field frequency $\omega$ now refers to the average field frequency; strictly speaking, the notion of a photon looses its meaning.

Our treatment of the interaction of helium with such a pulse is based on the solution of the time-dependent Schrödinger equation by means of a spectral method of configuration interaction type. It consists of expanding the wave function of helium in a basis of products of $L^{2}$-integrable functions of the electron radial coordinates and bipolar harmonics of the angular coordinates. In the present case, the $L^{2}$-integrable functions are either Coulomb-Sturmian functions [6] or B-spline functions [25]. After the end of the pulse, the information about the differential TPDI probability densities are obtained 
by projecting either the total or the doubly ionized wave packet on a symmetrized product of two Coulomb wave functions of effective charge $Z$. The doubly ionized wave packet is extracted by subtracting, from the total wavepacket, its bound state and single continuum components. The latter component is obtained by means of the J-matrix method. This method allows to calculate the multichannel single continuum scattering wave function for a given input channel $\Gamma$ characterized by a set of parameters including the quantum numbers of the inner electron, the angular momentum of the outer electron and the total angular momentum of the system and its projection along the quantization axis. This function writes:

$$
\Theta_{\Gamma}\left(E, \vec{r}_{1}, \vec{r}_{2}\right)=\sum_{j} b_{j}^{\Gamma}(E) \Psi_{j}\left(\vec{r}_{1}, \vec{r}_{2}\right)+\Phi_{\Gamma}\left(\vec{r}_{1}, \vec{r}_{2}\right)
$$

where $E$ is the total energy of the system and $\vec{r}_{1}, \vec{r}_{2}$, the position vectors (with respect to the nucleus supposed to be infinitely massive) of electron 1 and 2 respectively. The functions $\Psi_{j}$ are the He bound states and pseudostates generated by diagonalizing the total hamiltonian in our finite basis of Coulomb-Sturmian functions [6]. The function $\Phi_{\Gamma}$ describes the outer region part of the wavefunction. It is expressed in terms of an infinite sum of products of Sturmian functions that reproduces the correct asymptotic behaviour for one electron ionized and the other one bound. Note that in all cases we examine below, both approaches lead to the same conclusions.

Let us first examine how the pulse duration affects the energy distribution of the escaping electrons. We take the average photon energy to be $50 \mathrm{eV}$, and the peak intensity of the pulse to be $10^{13}$ Watt $/ \mathrm{cm}^{2}$. We consider two total pulse durations, namely 10 optical periods and 3 optical periods. The results were obtained by projecting the total wave packet on a symmetrized product of two Coulomb wavefunctions of effective charge $Z=2$ at the end of the pulse. These Coulomb wavefunctions were normalized in momentum space, and the density-of-states factor, which is the product of the electron momenta, has been included in the results for the probability density. It is of course questionable whether a product of two Coulomb wavefunctions gives a meaningful description of the final state, particularly at short times where the electrons are still close to one another. However, this is the best we can do at the present stage. Further investigation, using a more accurate final-state wavefunction, is clearly warranted despite the formidable challenge it implies.

For a pulse duration of 10 optical periods (Figure 2) we recognize the usual energy distribution which has a U-shape along the energy conservation line $E_{1}+E_{2}=2 \hbar \omega-$ $79 \mathrm{eV}$. At a photon energy of $50 \mathrm{eV}$ dynamical screening is no longer crucial for both electrons to escape. (Note that performing the projection of the final wave packet on two Coulomb functions some time after the end of the pulse does not change the conclusions.) The photon energy is just $4.4 \mathrm{eV}$ below the threshold for sequential double ionization to occur, and since the pulse duration is much longer than the relaxation time one electron can absorb a photon well before the other and carry away most of the dielectronic interaction energy. Reducing the total pulse duration to 3 optical periods (Figure 3) has a dramatic effect: both electrons have a strong tendency to share equally 
Time-dependent analysis of the mechanism for two-photon double-escape in helium: from very long to attose

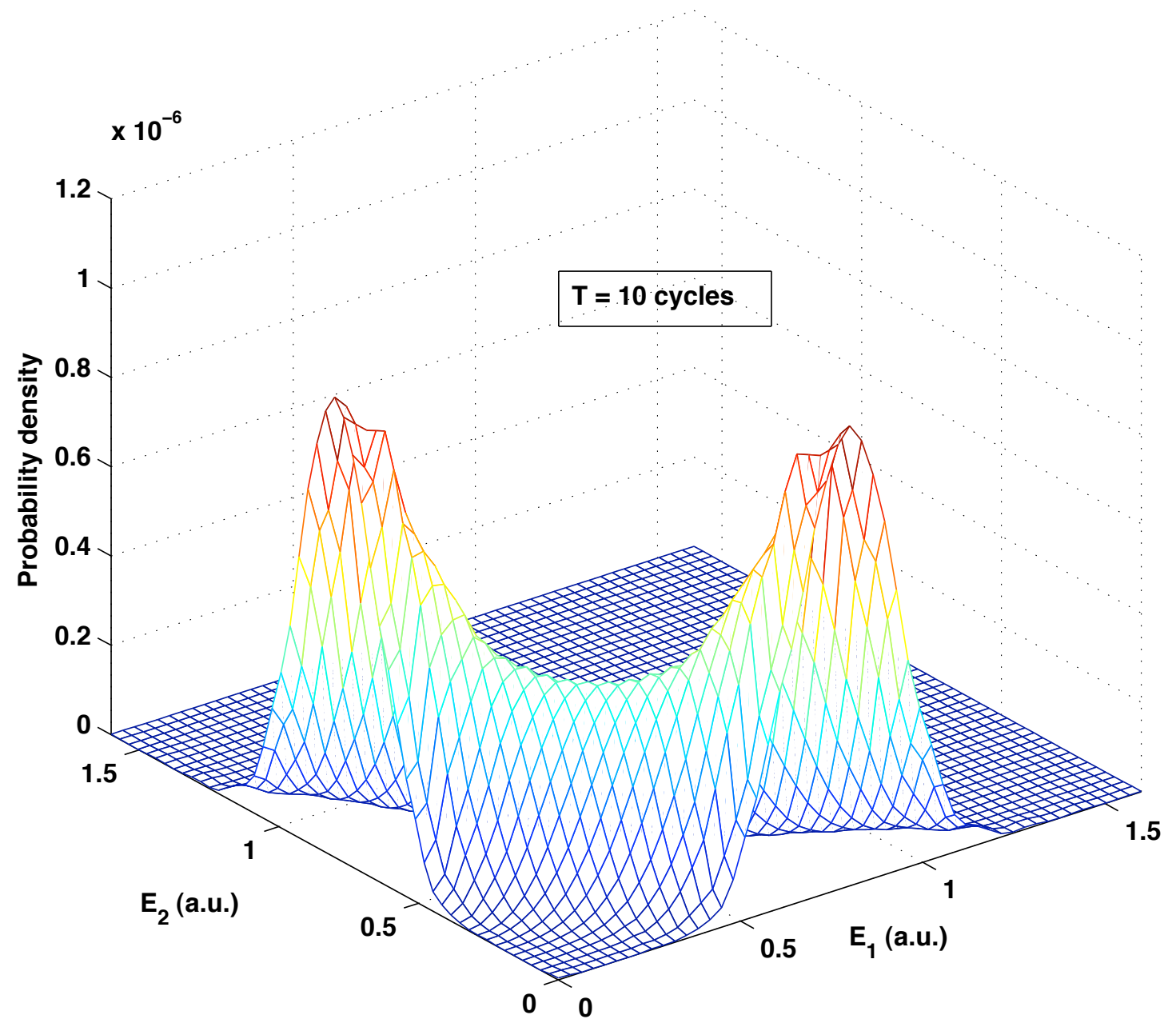

Figure 2. Differential probability density (in a.u.) with respect to the energy of the emergent electrons for TPDI of helium. We have integrated over the directions of the electrons. The pulse has a total duration of 10 optical cycles, the average photon energy is $50 \mathrm{eV}$, and the peak intensity is $10^{13} \mathrm{Watt} / \mathrm{cm}^{2}$.

the available energy. Now the pulse duration is not much larger than the relaxation time, and the pulse does not last long enough for one electron to absorb a photon well before the other.

A detailed analysis of the time evolution of the radial doubly ionized wave packet indicates that immediately after the end of the interaction with the pulse, both electrons have about the same energy and are at similar distances from the nucleus. In Figure 4 , we show snapshots of the doubly ionized wave packet at various instants after the pulse of 3 optical cycles has ceased to interact with the atomic system. We clearly see that at the end of the interaction with the pulse, the major part of the wave packet 
Time-dependent analysis of the mechanism for two-photon double-escape in helium: from very long to attose

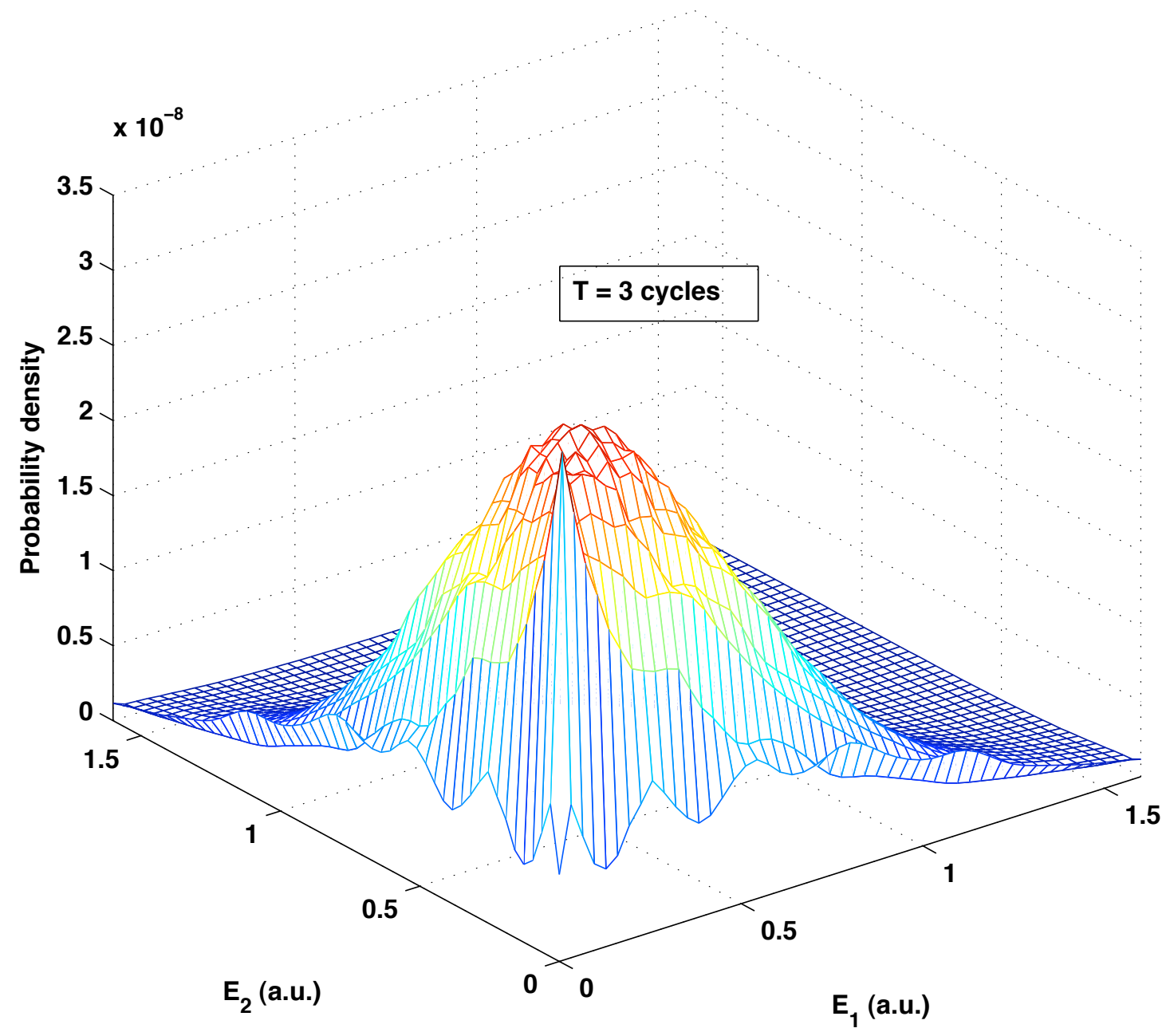

Figure 3. Same as Figure 2 but for a pulse duration of 3 optical cycles.

is already concentrated along the line $r_{1}=r_{2}$ showing that the screening is no longer dynamic but rapidly becomes static. This suggests that each electron more or less independently, and simultaneously, absorbs one "photon" (of average energy $50 \mathrm{eV}$ ) at very short time scales. Note that a photon with moderate energy carries negligible momentum (zero in the dipole approximation) so it is most probable that when TPDI occurs in this energy range each of the two photons is absorbed by a different electron; if both photons were to be absorbed by the same electron, that electron would have to transfer significant momentum to the other electron through a hard collision, which is unlikely. Although the individual electron angular momentum number $\ell_{i}$ is not a good quantum number, among the different $\left(\ell_{1}, \ell_{2}\right)$ configurations of the electron pair the $(1,1)$ configuration is the dominant one, in accord with our assertion that each electron absorbs one "photon". In order to gain more insight into the double escape 


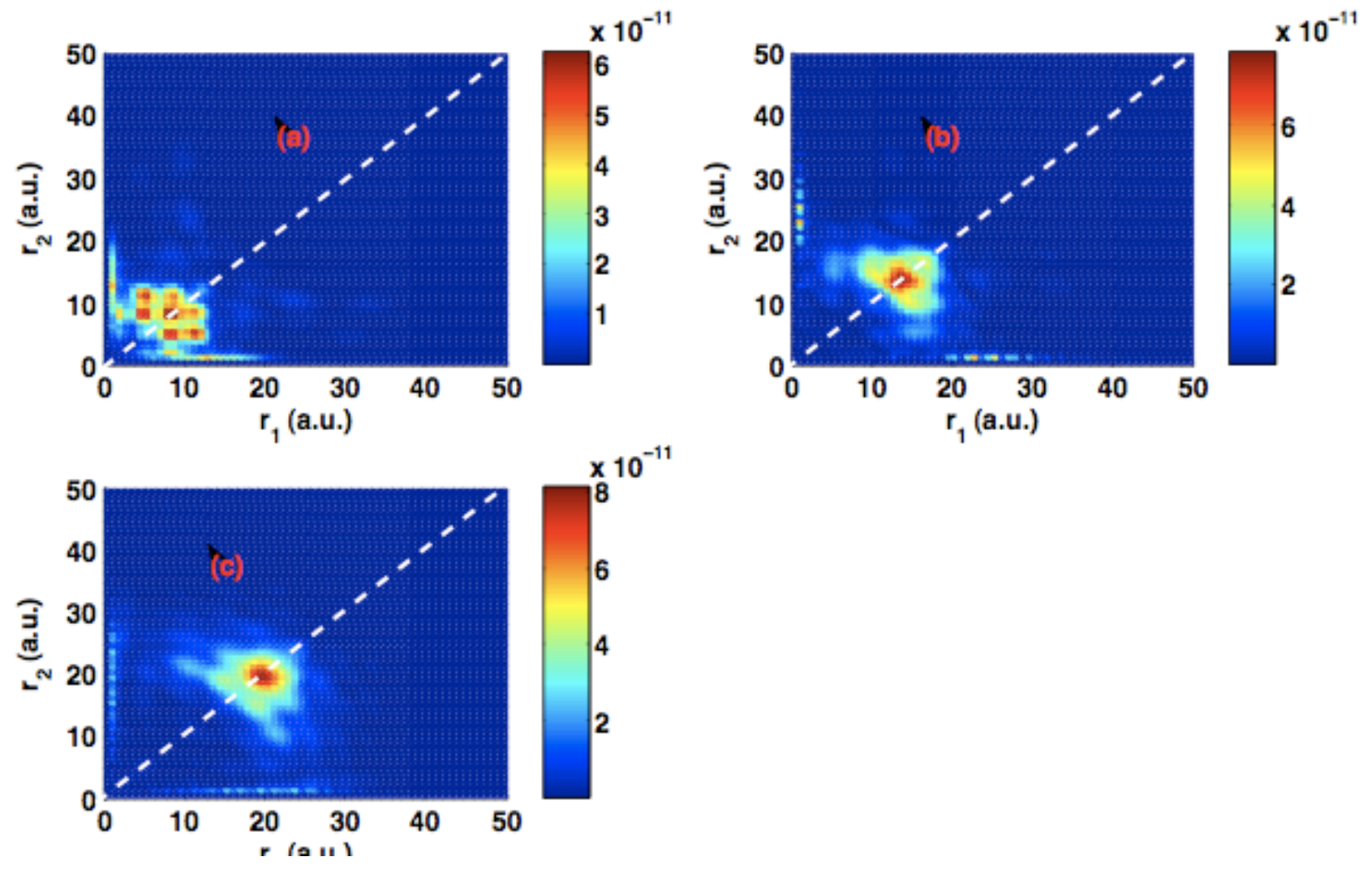

Figure 4. Snapshots of the probability density associated to the radial doubly ionized wave packet at different times: (a) at the end of the interaction of the pulse with the atomic system; (b) 1.5 optical period and (c) 3 optical periods after the pulse has ceased to interact with the atomic system. The pulse has a sine squared profile, its duration is 3 optical periods, the photon energy is $50 \mathrm{eV}$, and the peak intensity is $10^{13} \mathrm{Watt} / \mathrm{cm}^{2}$.

mechanism, let us examine the angular distributions. We consider a pulse with peak intensity $10^{13}$ Watt $/ \mathrm{cm}^{2}$ and an average photon energy of $50 \mathrm{eV}$, as before, but now we reduce the duration to two cycles. We consider the case where the electrons have the same energy, namely, $10.5 \mathrm{eV}$. The calculations were performed by projecting the doubly ionized wave packet onto a symmetrized product of two Coulomb wavefunctions with effective charge $Z=2$. We suppose that one of the electrons is emitted along $\mathrm{O} z$, the polarization axis, while the other one is detected at an angle $\theta$ with respect to $\mathrm{O} z$. If the projection of the doubly ionized wave packet onto the symmetrized product of Coulomb functions is performed just at the end of the interaction with the pulse (blue line on Figure 5) we see that the probability for both electrons to escape in the same direction is the same as the probability for them to escape in opposite directions. The reason that these two probabilities are the same is that each electron absorbs a photon more or less independently of the other, and a given electron is as likely to emerge parallel as antiparallel to the polarization axis. Accordingly, the $(1,1)$ configuration is the dominant one in the full wave packet. However, after photoabsorption occurs, and 
Time-dependent analysis of the mechanism for two-photon double-escape in helium: from very long to attose

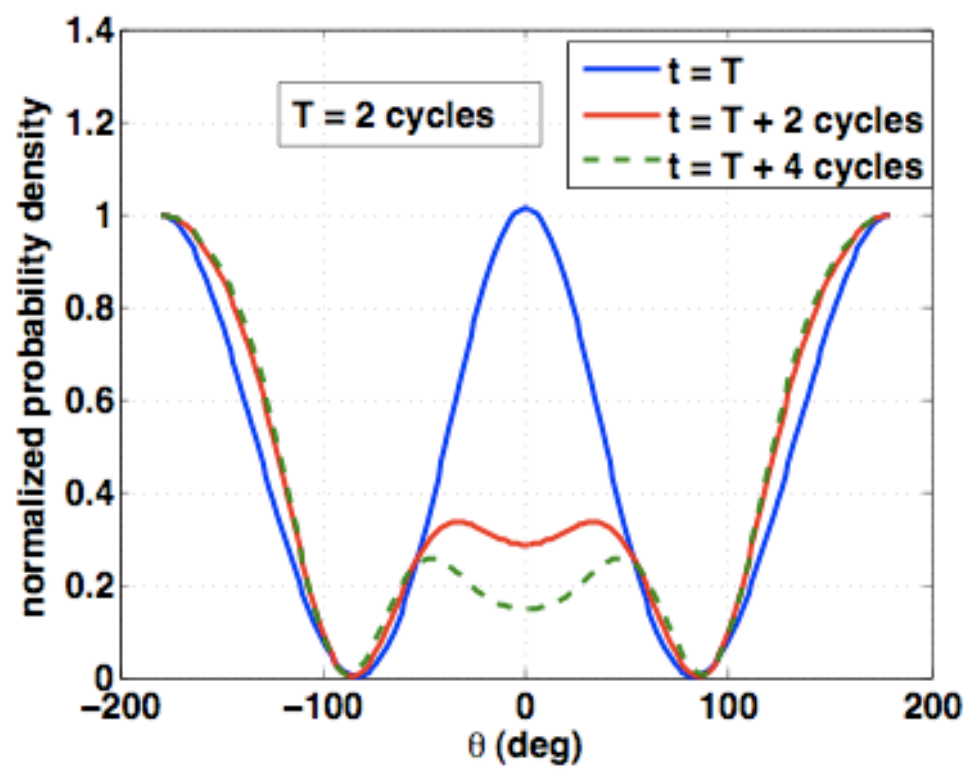

Figure 5. Normalized triply differential probability density in a.u. for TPDI of helium by a cosine-squared pulse of duration $T=2$ optical periods, average photon energy 50 $\mathrm{eV}$, and peak intensity $10^{13} \mathrm{Watt} / \mathrm{cm}^{2}$. Both electrons have an energy of $10.5 \mathrm{eV}$. One electron escapes along $\mathrm{O} z$, the polarization axis, while the second electron is detected at an angle $\theta$ with respect to $\mathrm{O} z$. The blue line is the result obtained by projecting the doubly ionized wave packet on a symmetrized product of Coulomb functions with $Z=2$ at the time $t=T$ when the interaction with the pulse end. The red and dashed lines, respectively, are the results obtained when the projection is made at a time $t$ which is 2 and 4 optical periods later.

the electrons begin their escape, their motion is more strongly correlated. In particular, if the electrons emerge in the same direction their motion is unstable since the electrons repel each other. Indeed, if the projection is performed 2 optical periods after the end of the pulse (red line in Figure 5) we clearly see that the probability for both electrons to escape in the same direction drops dramatically. An analysis of the angular-momentum pairs reveals that the $(0,2)$ configuration becomes significant and starts to interfere destructively with the $(1,1)$ configuration. In physical terms, if the electrons emerge in the same direction their speeds differ, if only infinitesimally, and the slower electron eventually reverses course since it is pushed by the faster electron towards the nucleus, to which it is also attracted. Our results show that angular configuration mixing, i.e. angular correlations, starts to appear after about 2 optical periods. The effect is even more important 4 optical periods after the end of the pulse: more configurations become important and we practically recover back-to-back emission. In fact the growth of 
Time-dependent analysis of the mechanism for two-photon double-escape in helium: from very long to attose

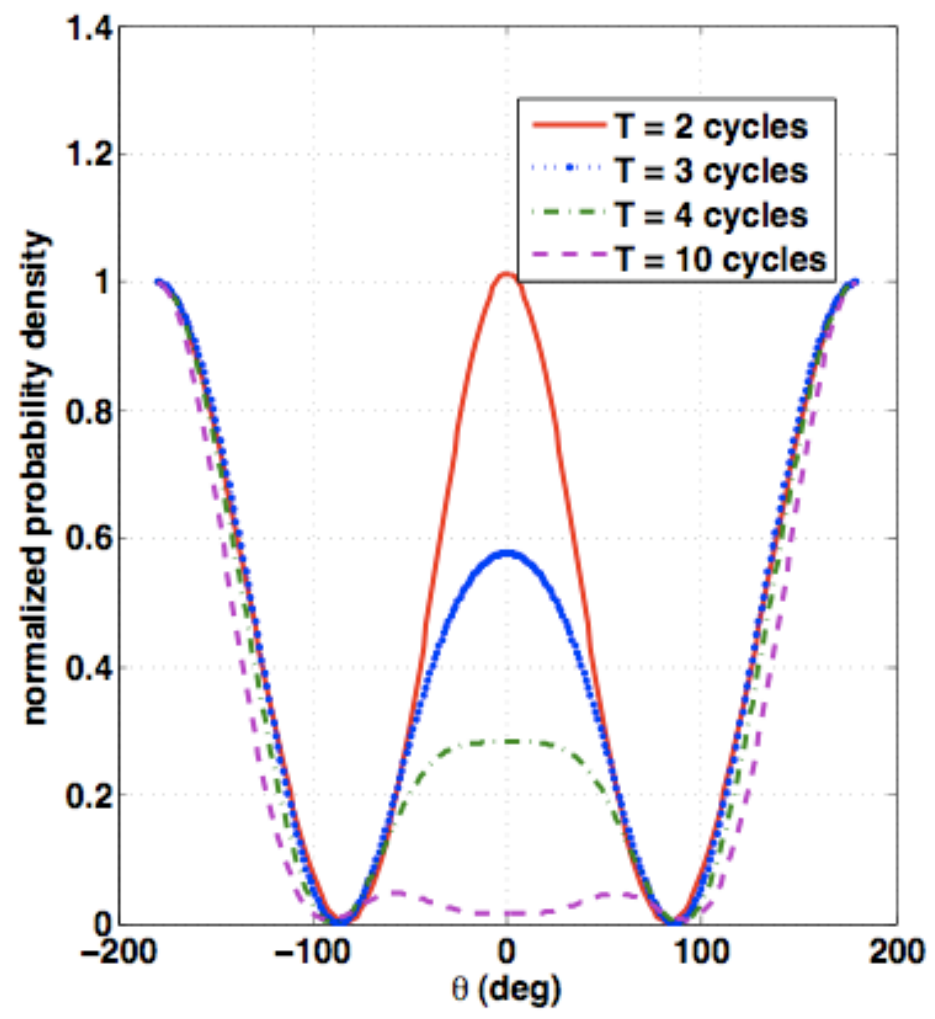

Figure 6. Same as Figure 5, but for various pulse durations $T$. As before the doubly ionized wave packet is projected onto a symmetrized product of Coulomb functions with $Z=2$, but now this projection is performed only at the end of the interaction with the pulse. The pulse durations considered are: 2 optical periods (red line), 3 optical periods (blue line), 4 optical periods (green dot-dashed line) and 10 optical periods (mauve dashed line).

individual angular momenta is unlimited, as explained many years ago by Popov and Benayoun [26] and Gailitis [27] using classical mechanics, an effect which seems to have been overlooked in the literature (but which, incidentally, may have been seen recently by Feist et al. [12]). Gailitis observed that the angular momentum of the first electron satisfies the equation $\dot{\vec{\ell}}_{1}=\vec{r}_{1} \times \vec{F}_{12}$. At a large time $t$, we have $r_{1} \sim t$ and the Coulomb force between the electrons is $F_{12} \sim t^{-2}$. As a result, $\dot{\ell}_{1} \sim \sin \left(\theta_{1}\right) t^{-1}$ and the integration gives $\ell_{1} \sim \sin \left(\theta_{1}\right) \ln (t)+$ a constant where $\theta_{1}$ is the angle between $\vec{r}_{1}$ and $\vec{r}_{12}$. This clearly shows that the value of $\ell_{1}$ increases logarithmically with time except when the torque vanishes, as it does if both electrons move along the same direction $\left(\theta_{1}=0\right.$ or $\pi)$, which is predominantly the case here. Note that this results holds also for $\ell_{2}$ but the total angular momentum remains finite. 
Time-dependent analysis of the mechanism for two-photon double-escape in helium: from very long to attose

Our results for the angular distribution demonstrate that there is a time scale associated to the electron-electron correlations. In the present case, it takes at least 100 as for electron correlations to manifest. This is confirmed by the results given in Figure 6 where the normalized triply differential probability density is shown for various total durations of the pulse with the same peak intensity and average photon energy as in the previous case. The normalized probability for back-to-back emission is roughly the same whether it is calculated just after the interaction of He with a four-cycle pulse or two optical cycle after the end of the interaction of He with a two-cycle pulse.

The previous discussion shows that, in general, after a sufficiently long time, the $(1,1)$ and $(0,2)$ configurations are mixed, and it is no longer possible to disentangle the process where each electron absorbs one photon from the process where one electron absorbs both photons and subsequently ejects the second electron by collision, even though the latter process is not expected to play a significant role. There are, however, two limiting situations where it is possible to clearly estimate the weight of each of these processes. The first situation concerns a purely sequential transition in the long pulse regime. In that case, each electron absorbs one photon and TPDI occurs through transition channels that require no electronic correlation. As a result, the $(1,1)$ configuration is the only one that plays a role. In the second situation, discussed in detail in the following section, the helium atom interacts with an ultrashort pulse.

\section{The ultrashort pulse duration limit}

In order to study the interaction of helium with an ultrashort pulse we calculate the TPDI amplitude within lowest order time-dependent perturbation theory. Using eq. (1) for the electric field, the transition probability amplitude in the length gauge is

$$
\begin{aligned}
& U_{f i}^{(2)}\left(\frac{\pi \tau}{2},-\frac{\pi \tau}{2}\right)=\frac{E_{0}^{2}}{4} \sum_{\ell}\left\langle\psi_{f}\left|z_{1}+z_{2}\right| \psi_{\ell}\right\rangle\left\langle\psi_{\ell}\left|z_{1}+z_{2}\right| \psi_{i}\right\rangle \times \\
& \quad\left(K\left(\omega, \omega, E_{\ell}\right)-K\left(\omega,-\omega, E_{\ell}\right)-K\left(-\omega, \omega, E_{\ell}\right)+K\left(-\omega,-\omega, E_{\ell}\right)\right),
\end{aligned}
$$

where $\left|\psi_{i}\right\rangle,\left|\psi_{f}\right\rangle$, and $\left|\psi_{\ell}\right\rangle$ represent the initial, final, and intermediate states, respectively, of helium. Note that the summation over $\ell$ includes an integration over the energy of the intermediate continuum states. The electron position vectors $\vec{r}_{1}$ and $\vec{r}_{2}$ have $z$-components $z_{1}$ and $z_{2}$, respectively, and $K(\omega, \bar{\omega})$ is the kernel given by:

$$
\begin{aligned}
K\left(\omega, \bar{\omega}, E_{\ell}\right)=\int_{-\frac{\pi \tau}{2}}^{\frac{\pi \tau}{2}} \mathrm{~d} \xi_{1} \cos ^{2}\left(\frac{\xi_{1}}{\tau}\right) \mathrm{e}^{i\left(\omega+\omega_{f \ell}\right) \xi_{1}} \times \\
\\
\int_{-\frac{\pi \tau}{2}}^{\xi_{1}} \mathrm{~d} \xi_{2} \cos ^{2}\left(\frac{\xi_{2}}{\tau}\right) \mathrm{e}^{i\left(\bar{\omega}+\omega_{\ell i}\right) \xi_{2}},
\end{aligned}
$$

where $\omega_{i j}=E_{j}-E_{i}$, namely the difference between the energies of the states represented by $\left|\psi_{j}\right\rangle$ and $\left|\psi_{i}\right\rangle$. The calculation of the kernel is straightforward though quite tedious. In the limit where the pulse duration is infinite, the kernel is a distribution that involves a delta function and a principal part for the integration over the energy of the intermediate continuum states in eq. (4). By contrast, in the limit of interest here, where the pulse 
duration is vanishingly small, the kernel is a very smooth quasi-constant function of the energy of the intermediate states. Under these conditions we can replace the energies of the intermediate states by an averaged value $\bar{E}_{\ell}$ and then use the closure relation to perform the summation over the intermediate states; we refer to this simplification as the "Slowly Varying Kernel Approximation" (SVKA). We stress that the SVKA is not the closure approximation. The latter has been used by Kheifets et al. [28] to calculate the total TPDI cross section within time-independent perturbation theory where there is no real criterion for choosing the average intermediate state energy $\bar{E}_{\ell}$. By contrast, for ultrashort pulse durations, i.e. for sufficiently small values of $\tau$, the choice of $\bar{E}_{\ell}$ is irrelevant because the kernel is very smooth and almost constant. In our calculations, we put $\bar{E}_{\ell}=E_{i}+\omega$. In addition, among the four terms between brackets in eq. (4), by far the dominant one is $K\left(-\omega,-\omega, E_{\ell}\right)$; this term corresponds to the absorption of two photons of average frequency $\omega$. As a result, the transition probability amplitude reads:

$$
\begin{aligned}
U_{f i}^{(2)}\left(\frac{\pi \tau}{2},-\frac{\pi \tau}{2}\right) \approx \frac{E_{0}^{2}}{4} K & \left(-\omega,-\omega, \bar{E}_{\ell}\right) \\
& \left(\left\langle\psi_{f}\left|z_{1}^{2}+z_{2}^{2}\right| \psi_{i}\right\rangle+2\left\langle\psi_{f}\left|z_{1} z_{2}\right| \psi_{i}\right\rangle\right) .
\end{aligned}
$$

The first term, the one proportional to $\left\langle\psi_{f}\left|z_{1}^{2}+z_{2}^{2}\right| \psi_{i}\right\rangle$, describes the process in which one electron absorbs two photons while ejecting the second electron by a collision. The second term, the one proportional to $\left\langle\psi_{f}\left|z_{1} z_{2}\right| \psi_{i}\right\rangle$, describes the process in which each electron absorbs one photon. In order to estimate the weight of these terms, we have performed a very simple calculation in which we choose the initial- and final-state wavefunctions to be

$$
\begin{aligned}
& \left\langle\vec{r}_{1}, \vec{r}_{2} \mid \psi_{i}\right\rangle=\frac{Z_{e}^{3}}{\pi} \mathrm{e}^{-Z_{e}\left(r_{1}+r_{2}\right)}, \text { with } Z_{e}=27 / 16 \\
& \left\langle\vec{r}_{1}, \vec{r}_{2} \mid \psi_{f}\right\rangle=\frac{1}{\sqrt{2}}\left(\psi_{\vec{k}_{1}}^{(-)}\left(\vec{r}_{1}\right) \psi_{\vec{k}_{2}}^{(-)}\left(\vec{r}_{2}\right)+\psi_{\vec{k}_{2}}^{(-)}\left(\vec{r}_{1}\right) \psi_{\vec{k}_{1}}^{(-)}\left(\vec{r}_{2}\right)\right),
\end{aligned}
$$

where $\psi_{\vec{k}}^{(-)}(\vec{r})$ is a Coulomb function of effective charge $Z=2$ with an asymptotic incoming wave behaviour. We find that the second term of the expression between brackets in eq. (6) is the dominant one. More quantitatively, the contribution of the second term to the energy distribution is five times higher than the first one in the case of a two-cycle pulse and a photon energy of $50 \mathrm{eV}$. This is true irrespective of the energy partition of the electrons. We conclude that the process in which each electron absorbs one photon dominates on very short time scales. We have also calculated the total TPDI probability as a function of the average photon energy for a pulse duration of 2 optical cycles, and peak intensity $10^{13} \mathrm{Watt} / \mathrm{cm}^{2}$. Note that the absolute duration of the pulse decreases with increasing average photon energy. In Figure 7, we compare the results (open triangles) obtained using the SVKA with results obtained from three different calculations employing the time-dependent Schrödinger equation. In the first of these latter calculations we used the J-matrix technique, while in the other two calculations we projected the total wave packet at the end of the pulse onto a symmetrized product of Coulomb functions with either $Z=2$ or $Z=1.75$. As expected, the SVKA results are in excellent agreement with the results obtained by projecting the total wave packet at 
Time-dependent analysis of the mechanism for two-photon double-escape in helium: from very long to attose

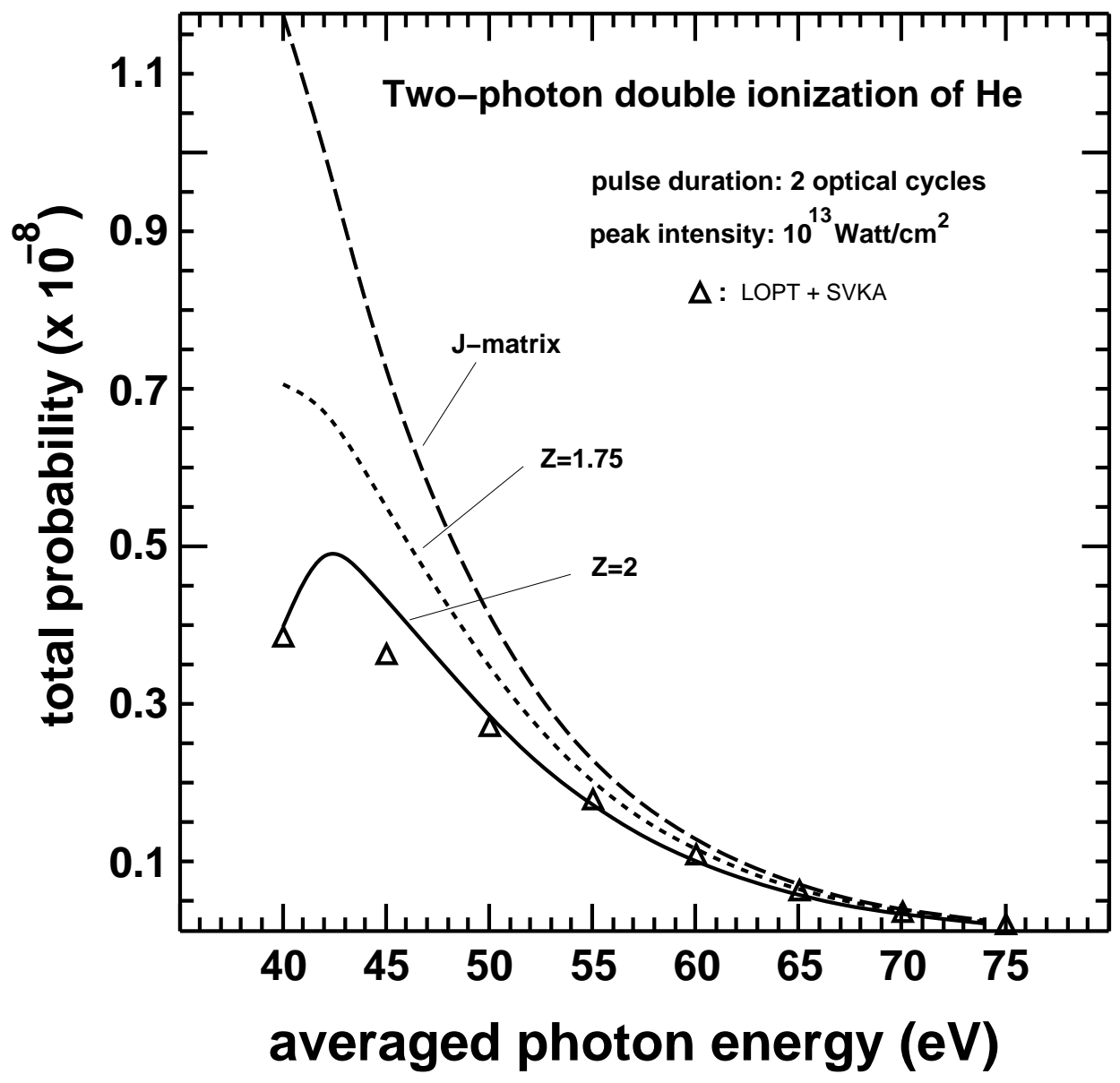

Figure 7. Total TPDI probability as a function of the averaged photon energy in eV for a pulse of $10^{13}$ Watt $/ \mathrm{cm}^{2}$ peak intensity and of 2 optical cycles, total duration. The full line is obtained by projecting the total wave packet, at the end of the pulse, on a symmetrized product of two Coulomb wave functions of $Z=2$. This result compares very well with those (open triangles) obtained within the lowest order time-dependent perturbation theory and the SVKA. The dotted line is obtained by projecting the total wave packet on Coulomb functions of $Z=1.75$. The dashed line corresponds to J-matrix calculations.

the end of the pulse on two Coulomb functions of effective charge $Z=2$. The agreement becomes perfect at very high photon energies, which is not surprising since, in principle, the SVKA becomes exact when the absolute duration of the pulse tends to zero (at an average photon energy of $65 \mathrm{eV}$, for instance, the absolute duration of the pulse is 140 as). The reason that the two calculations based on the J-matrix and on the projection of the total wave packet onto Coulomb functions with $Z=1.75$ do not approach zero at an average photon energy of $39.5 \mathrm{eV}$, i.e. at the double-ionization threshold, is that this threshold is not defined any more. Note that Coulomb functions with $Z=1.75$ are not orthogonal to the wave functions associated to the bound states of $\mathrm{He}^{+}$. Nevertheless, It is interesting that the two curves are very far from each other and from the $Z=2$ curve suggesting that the screening effect we discussed above does indeed play an important 
Time-dependent analysis of the mechanism for two-photon double-escape in helium: from very long to attose role.

\section{Conclusions}

In this contribution we presented a numerical simulation of the interaction of helium with an ultrashort pulse in the attosecond regime. Our purpose was to gain insight into the actual mechanism of two-photon double-ionization of helium by analyzing this process on very short time scales. We considered the case of a pulse whose averaged photon energy is $50 \mathrm{eV}$. Our simulations underline the following two effects: (i) the shape of the energy distribution changes dramatically from a U-shape for long pulses to an inverse U-shape for short pulses and (ii) the angular correlations require a finite time before manifesting. A deeper analysis shows that over very short time scales, the contribution of the $(1,1)$ configuration is much more important than the $(0,2)$ configuration. If, as seems reasonable, we associate with the $(1,1)$ configuration the process in which each electron absorbs one photon, our results show that it is this process which dominates at short time scales. Electron correlations are unnecessary for double escape to occur. However, if we let the system evolve after the pulse or if we consider longer pulses, configuration mixing occurs and leads to back-to-back emission. Thus at longer times the $(1,1)$ and $(0,2)$ configurations are entangled, and this masks the primary mechanism for ionization since the $(0,2)$ configuration is associated with the process in which the same electron absorbs both photons. In order to confirm this interpretation, we treated this problem within the lowest order of time-dependent perturbation theory. In the limit of ultrashort pulses, we showed that within the slowly varying kernel approximation, it is indeed possible to separate the contributions of the two different processes in a non ambiguous way. It is important to note that in the present frequency regime, these conclusions stay the same irrespective of the theoretical approach we use to calculate the two-photon double-ionization probability. In addition, the present conclusions complement our previous interpretation of the double escape mechanism.

\section{Acknowledgments}

The authors wish to thank Yuri Popov for very interesting discussions. HB and RS thank the Laboratoire de Physique Atomique, Moléculaire et Optique (unité PAMO) of the Université catholique de Louvain for hospitality and financial support. The authors thank the Université catholique de Louvain for providing them with an access to the supercomputer of the CISM (Calcul Intensif et Stockage de Masse) which is supported by the FNRS through the FRFC (Fonds de la Recherche Fondamentale et Collective) project no 2.4556.99, "Simulations Numériques et traitement des données". 
Time-dependent analysis of the mechanism for two-photon double-escape in helium: from very long to attose

\section{References}

[1] Dörner R, Feagin J M, Cocke C L, Bräuning H, Jagutzki O, Jung M, Kanter E P, Khemliche H, Kravis S, Mergel V, Prior M H, Schmidt-Böcking H, Spielberger L, Ullrich J, Unversagt M and Vogt T 1996 Phys. Rev. Lett. 77, 1024

[2] Bräuning H, Dörner R, Cocke C L, Prior M H, Krässig B, Kheifets A S, Bray I, Bräuning-Demian A, Carnes K, Dreuil S, Mergel V, Richard P, Ullrich J and Schmidt-Böcking J. Phys. B: At. Mol. Opt. Phys. 315149

[3] Laulan S and Bachau H 2003 Proc. Int. Conf. on Electron and Photon Impact Ionization and Related Topics, Metz, France (IOP Conf. Ser. No. 172) ed L U Ancarani p 109

[4] Feng L and van der Hart H W 2003 J. Phys. B: At. Mol. Opt. Phys. 36 L1

[5] Hu S X, Colgan J and Collins L A 2005 J. Phys. B: At. Mol. Opt. Phys. 38 L35

[6] Foumouo E, Lagmago Kamta G, Edah G and Piraux B 2006 Phys. Rev. A 74063409

[7] Horner D A, Morales F, Rescigno T N, Martin F and McCurdy C W 2007 Phys. Rev. A 76030701

[8] Nikolopoulos L A A and Lambropoulos P 2007 J. Phys. B: At. Mol. Opt. Phys. 401347

[9] Ivanov I A, Kheifets A S 2007 Phys. Rev. A 75033411

[10] Shakeshaft R 2007 phys. Rev. A 76063405

[11] Sorokin A A, Wellhöfer M, Bobashev S V, Tiedke K and Richter M 2007 Phys. Rev. A75 051402(R)

[12] Feist J, Nagele S, Pazourek R, Persson E, Schneider B I, Collins L A and Burgdörfer 2008 Phys. Rev. A 77043420

[13] Antoine Ph, Foumouo E, Piraux B, Shimizu T, Hazegawa H, Nabekawa Y and Midorikawa K 2008 Phys. Rev. A $\mathbf{7 8} 023415$

[14] Rudenko A, Foucar L, Kurka M, Ergler Th, Kühnel K U, Jiang Y H, Voitkiv A, Najjari B, Kheifets A, Lüdemann S, Havermeier T, Smolarski M, Schössler S, Cole K, Schöffler M, Dörner R, Düsterer S, Li W, Keitel B, Treusch R, Gensch M, Schröter C D, Moshammer R and Ullrich J 2008 Phys. Rev. Lett. 101, 073003

[15] Palacios A, Rescigno T N and McCurdy C W 2009 Phys. Rev. A 79033402

[16] Foumouo E, Antoine Ph, Piraux B, Malegat L, Bachau H and Shakeshaft R 2008 J. Phys. B 41 051001

[17] Ayvazyan et al. 2006 Eur. Phys. J. D 37297

[18] Balcou Ph, Salière P, Budil K S, Ditmire T, Perry M D and L'Huillier 1995 Z. Phys. D 34107

[19] Bachau H and Lambropoulos P 1991 Phys. Rev. A 44 R9

[20] Lambropoulos P, Nikolopoulos L A A, Makris M G and Mihelic A 2008 Phys. Rev. A 78055402

[21] Foumouo E, Antoine Ph, Bachau H and Piraux B 2008 New J. Phys. 10025017

[22] Feist J, Nagele S, Pazourek R, Persson E, Schneider B I, Collins L A and Burgdörfer 2009 J. Phys. B:At. Mol. Opt. Phys. 42134014

[23] If one places two electrons at equal distances $r$ on opposite sides from a doubly-charged nucleus, then the potential would be given by $-2 / \mathrm{r}-2 / \mathrm{r}+1 /(2 \mathrm{r})=-2 \mathrm{Z} / \mathrm{r}$ where $\mathrm{Z}$ is the effective charge. The solution of this equation gives $\mathrm{Z}=1.75$.

[24] Rau A R P 1984 Comments At. Mol. Phys. 14285

[25] Bachau H, Cormier E, Decleva P, Hansen J E and Martín F 2001 Rep. Prog. Phys. 641815

[26] Popov Yu V and Benayoun J J 1981 J. Phys. B 143513

[27] Gailitis M 1990 J. Phys. B 2385

[28] Kheifets A S and Ivanov I A 2006 J. Phys. B:At. Mol. Opt. Phys. 391731 